In the book which accompanies the stencils various methods of finding quadratic residues are discussed and the method by means of the expansion of the square root of the number in a continued fraction is found to be by far the most effective. Various examples are given illustrating the power of the stencils, and a reproduction of the first page of the list of primes accompanies the work.

The plan of the stencils was first conceived in November 1924 and has been carried on since then under grants from the Carnegie Institution of Washington. Plans for the distribution of the sets are not yet completed, but every effort will be made to place them where they will be of most use.

The University of California

\title{
ON A PROBLEM IN THE THEORY OF GROUPS ARISING IN THE FOUNDATIONS OF INFINITESIMAL GEOMETRY*
}

BY H. P. ROBERTSON AND H. WEYL

In another paper in this issue, $\nmid$ the fundamental problem of infinitesimal geometry is formulated as the problem of uniquely associating with an arbitrary coordinate system on the manifold $M$ a normal coordinate system on the tangent plane $T_{P}$ by means of the fundamental coefficients of displacement on $M$.

The importance of the other aspect of this problem raised by $\mathrm{O}$. Veblen and $\mathrm{H}$. P. Robertson, yet remains: to associate a transformation of the given group (5) with an arbitrary tranformation of the coordinates $x$ in such a way that it gives rise to a representation by (B), that is, that to composition of arbitrary transformations of $x$ corresponds composition of the associated transformations of (5). From

* Presented to the Society, June 21, 1929.

$\dagger$ This issue, pp. 716-725. 
this standpoint the problem is a pure group-theoretic one which is not at all concerned with a "connection" on $M$. We consider in the following the projective group as an example.

More precisely, the problem is the following. Take the point $P$ under consideration as the origin of coordinates, that is, consider the relative coordinates $x_{k}-x_{0}{ }^{i}$, where $P=P\left(x_{0}\right)$. An arbitrary transformation of these relative coordinates $x^{i}$ has the form

$$
s: \quad \bar{x}^{i}=a_{k}^{i} x^{k}+\frac{1}{2} a_{k l}^{i} x^{k} x^{l}+\cdots .
$$

The associated $n$-dimensional projective transformation $\sigma$, which shall also leave the origin fixed, is expressed in homogeneous coordinates $\eta$ in accordance with the normalization (4) of the paper mentioned above. We take as coefficients $a_{k}^{i}$ of $\sigma$ the coefficients $a_{k}^{i}$ of (1); the coefficients $a_{k}$ are then to be determined. The condition imposed is that the association $s \rightarrow \sigma$ shall yield a representation. We may add the additional condition that $\sigma$ depend only on the coefficients $a_{k}{ }^{i}$ and $a_{k l}^{i}$ of $s$ of first and second orders.

We are then naturally led to the problem of representing the group (\$) of all transformations by linear transformations, whereby the accessory condition that the transformation corresponding to $s$ shall depend on the terms of the first two or first three orders etc. may be imposed. This problem is to be attacked as follows. We first restrict ourselves to the subgroup $B_{0}$ of $(S)$ consisting of linear transformations

$$
\bar{x}^{i}=a_{k}^{i} x^{k} .
$$

We know the representations of $\$_{0}$ and we shall assume that the given representation $s \rightarrow \sigma$ of $\$ s$ by $N$-dimensional matrices $\sigma$ decomposes into known irreducible representations when considered as a representation of $B_{0}$ (that is certainly the case when we restrict ourselves to the unimodular transformations of $\left(S_{0}\right.$; we take it to be the case for the full linear group for the sake of simplicity). Let these irreducible constituents be of orders $o, o^{\prime}, \cdots$, respectively; i.e. to the infinitesimal dilatation $d x^{i}=x^{i}$ correspond the dilatations 
$d \xi^{\iota}=o^{\iota}, d \xi^{\iota}=o^{\prime} \xi^{\iota}, \cdots$, respectively in these representations. (It follows from the general theory that in an irreducible representation a dilatation must correspond to a dilatation in the original group.)

Furthermore, we employ Lie's infinitesimal method. To an infinitesimal transformation $s$

$$
d x^{i}=a_{k}^{i} x^{k}+\frac{1}{2 !} a_{k l}^{i} x^{k} x^{l}
$$

corresponds an infinitesimal transformation

$$
d \xi^{\imath}=\alpha_{\kappa}{ }^{\imath} \xi^{\kappa}
$$

of the given representation. But the $\alpha_{\kappa}^{i}$ are now linear homogeneous functions of the $a_{k}{ }^{i}, a_{k l}^{i}, \cdots$. The condition that $\sigma$ be a representation requires in addition that to the commutator of two transformations $s$ corresponds the commutator of the corresponding transformations $\xi$. We consider in particular the two transformations

(4) $s$ : $\quad d x^{i}=\frac{1}{2 !} a_{k l} l^{i} x^{k} x^{l}+\cdots$ and $C$ : $\quad d^{\prime} x^{i}=c_{k}^{i} x^{k}$.

If the matrices of the corresponding $\sigma$ (3) be $\mathrm{A}$ and $\Gamma$, the infinitesimal $\sigma$ with matrix $\mathrm{A} \Gamma-\Gamma \mathrm{A}$ shall correspond to the commutator of (4). We proceed in three steps, by first taking as $C$ the dilatation

$$
d^{\prime} x^{i}=x^{i}, \text { that is, } c_{k}^{i}=\delta_{k}^{i},
$$

then the more general linear "principal transformation" $d^{\prime} x^{i}=\lambda_{i} x^{i}$ (not summed!) with arbitrary $\lambda_{i}$, and finally the unrestricted $C$.

The commutator of (2) and the dilatation is obtained by multiplying the term of $n$th degree in $(2)$ by $(n-1)$. The set of variables $\xi^{\imath}$ is divided into subsets the members of which are transformed among themselves under $\Gamma$, and in particular are only multiplied by $o, o^{\prime}$ etc. by the transformation $\Gamma=\Gamma_{d}$ which corresponds to the dilatation. The rectangle $[\Lambda]$ of the matrix $A$, in which the set $o$ intersects the 
set $o^{\prime}$, is multiplied by $o^{\prime}-o$ on going over to $\mathrm{A \Gamma}_{d}-\Gamma_{d} \mathrm{~A}$ from A. The $\alpha_{\kappa}{ }^{2}$ in this rectangle must consequently be multiplied by $o^{\prime}-o$ when the $a_{k}^{i}, a_{k l}^{i}$, etc. are replaced by $0 \cdot a_{k}^{i}, 1 \cdot a_{k l}^{i}$ etc. Consequently if $o^{\prime}-o$ is not a whole number, $0,1,2, \cdots,[\mathrm{A}]$ must vanish identically, and if $o^{\prime}-o=n-1$, [A] can depend only on the terms of order $n$ in (2). In particular the square [A] of A in which the set $o$ intersects itself can only depend on the $a_{k}^{i}$.

We can choose the coordinates in representation space in such a way that to the principal transformations $d x^{i}=\lambda_{i} x^{i}$ correspond principal transformations $\xi^{\iota} \rightarrow \Lambda_{\iota} \xi^{\iota}$; the "weights" $\Lambda_{\iota}$ are linear forms in the $\lambda_{i}$. In the commutator of (2) with this principal transformation $a_{k l}^{i}$ is replaced by

$$
a_{k l}^{i} \ldots\left(\lambda_{k}+\lambda_{l}+\cdots-\lambda_{i}\right)
$$

while the corresponding process for the $\sigma$ changes $\alpha_{\kappa}{ }^{2}$ into $\alpha_{\kappa}{ }^{\imath}\left(\Lambda_{\kappa}-\Lambda_{\imath}\right)$. Consequently the $\alpha_{\kappa}{ }^{\imath}$ can depend only on those $a_{k l}^{i} \cdots$ for which $\lambda_{k}+\lambda_{l}+\cdots-\lambda_{i}$ is identical with $\Lambda_{\kappa}-\Lambda_{\iota}$.

Equivalent representations, which differ only in the choice of the coordinate system in which they are expressed, shall naturally be considered as the same. But as soon as the irreducible representations into which the given representation of $\left(3_{0}\right.$ is decomposed is fixed the coordinate system is fixed except for multiplication of the variables of each subset by a non-vanishing arbitrary constant.

Let us return to the case considered above, in which $N=n+1$ and the $(n+1)$-dimensional representation of $\$_{0}$ is decomposed into the $n$-dimensional $s \rightarrow s$ and the one-dimensional which associates the identity $\xi^{0}=\xi^{0}$ with every $s$. Here $o=1, o^{\prime}=0$; we must consequently have in (3)

$$
\alpha_{k}^{i}=a_{k}^{i}, \quad \alpha_{0}^{0}=1, \quad \alpha_{0}^{i}=0,
$$

and $\alpha_{k}^{0}$ depends only on the terms $a_{r s}^{i}$ of second order. Furthermore, $\alpha_{k}^{0}$ must be linear form in those $a_{r s}^{i}$ only for which $\lambda_{r}+\lambda_{s}-\lambda_{i}=\lambda_{k}$. We must therefore have

$$
\alpha_{k}^{0}=\sum_{i} H_{i k} a_{i k}^{j}
$$


where the $H_{i k}$ are constants independent of $s$. In order to determine the $H_{i k}$ we now consider the commutator of

and obtain

$$
d x^{i}=\frac{1}{2} a_{k l}^{i} x^{k} x^{l} \text { and } d^{\prime} x^{i}=c_{k}^{i} x^{k}
$$

$$
\sum_{i, r} H_{i k}\left(a_{r k}^{i} c_{i}^{r}+a_{i r}^{i} c_{k}^{r}-a_{i k}^{r} c_{r}{ }^{i}\right)=\sum_{i, r}^{r} c_{k}^{r} H_{i r} a_{i r}^{i}
$$

or

$$
\sum_{i, r} a_{r k}^{i}\left(H_{i k}-H_{r k}\right) c_{i}{ }^{r}=\sum_{i, r} b_{k}^{r}\left(H_{i r}-H_{i k}\right) a_{i r}^{i} .
$$

Equating the coefficients of $c_{i}{ }^{k}$ on both sides for $i \neq k$ we see that $H_{i k}=H_{k k} ; H_{i k}$ does not depend on $i$ and can be written $H_{k}$. The equation then reduces to

$$
\sum\left(H_{r}-H_{k}\right) c_{k}^{r}=0
$$

which tells us that $H_{k}$ is independent of $k, H_{k}=H$. Hence

$$
\alpha_{k}^{0}=H \sum_{i} a_{i k}^{i} .
$$

When $H \neq 0$ we can multiply the variable $\xi^{0}$ by an appropriate constant in such a way that in this new coordinate system

$$
H=-\frac{1}{n+1} \text {. }
$$

We thus find that there are two distinct possibilities: (1) $\alpha_{k}^{0}=0$ or the affine tangent plane, (2) the one obtained by Robertson and, from another standpoint, in the paper by $\mathrm{H}$. Weyl on pages $716-725$ of this issue, that is, the semiosculating projective plane.

Princeton University 